\title{
The Protective Role of Klotho in CKD-Associated Cardiovascular Disease
}

\author{
Xianjin Bi Ke Yang Bo Zhang Jinghong Zhao \\ Department of Nephrology, The Key Laboratory for the Prevention and Treatment of Chronic Kidney Disease of \\ Chongqing, Kidney Center of PLA, Xinqiao Hospital, Army Medical University (Third Military Medical University), \\ Chongqing, China
}

\section{Keywords}

Chronic kidney disease $\cdot$ Cardiovascular diseases $\cdot$ Klotho

\begin{abstract}
Background: Cardiovascular diseases (CVDs) are the leading cause of morbidity and mortality in advanced CKD. The major pathological changes of CKD-associated CVD are severe vascular media calcification, aberrant cardiac remodeling such as hypertrophy and fibrosis, as well as accelerated atherosclerosis. a-Klotho is proposed as an anti-aging gene, which is primarily expressed in the kidney. Recent studies reveal that a-Klotho deficiency is associated with profound cardiovascular dysfunction. Of note, CKD represents extremely declined a-Klotho levels, hinting that a-Klotho deficiency may be implicated in the pathogenesis of CKD-associated CVD. Summary: Based on the pathogenic mechanism of a-Klotho deficiency and decreased Klotho levels in the circulation even early in stage 1 of CKD, a-Klotho serves as a sensitive biomarker for renal insufficiency and also a novel predictor of risk of overall mortality of CVD events in CKD. Meanwhile, loss of Klotho resulted from kidney dysfunction markedly contributes to the progressive development of CKD and CVD. By contrast, prevention of Klotho decline using exogenous supplementation or genetically activated
\end{abstract}

karger@karger.com www.karger.com/kdd

Karger $\frac{1}{\%}$
(C) 2020 The Author(s)

Published by S. Karger AG, Basel

This article is licensed under the Creative Commons AttributionNonCommercial-NoDerivatives 4.0 International License (CC BYNC-ND) (http://www.karger.com/Services/OpenAccessLicense) Usage and distribution for commercial purposes as well as any distribution of modified material requires written permission. ways by several mechanisms can dramatically mitigate cardiac dysfunction, prevent vascular calcification, and retard the progression of CKD-accelerated atherosclerosis. Key Messages: Klotho deficiency is proposed as a novel predictive biomarker as well as a pathogenic contributor to CVD events in CKD. In the future, Klotho may be a crucial potential therapeutic strategy to decrease the burden of CVD comorbidity with CKD in clinics.

(C) 2020 The Author(s)

Published by S. Karger AG, Basel

\section{Introduction}

CKD is a worldwide disease with a high incidence and prevalence, which puts a huge burden on public health. $\mathrm{CKD}$ is a progressive systemic disease, in which kidney structure and function are irreversibly damaged. Except for renal injury, CKD is always accompanied by multiple pathological changes of other organs, especially cardiovascular system [1]. Cardiovascular diseases (CVDs) are the primary cause of death in advanced CKD. The major pathological changes of CKD-associated CVD are severe vascular calcification (VC), aberrant cardiac remodeling with hypertrophy and fibrosis, and accelerated atherosclerosis [2]. Over the past decades, although consider- 
able efforts were devoted to investigate the etiology and pathogenesis involved in the progression of CVD associated with $\mathrm{CKD}$, the high-risk factor and its underlying mechanisms are still far from being elucidated.

The earliest discovered Klotho was named as a-Klotho, which was found in the animal experiment of primary hypertension by Kuro-o et al. [3]. Given its robust antiaging effect, $\alpha$-Klotho was initially considered as an antiaging gene. $\alpha$-Klotho is mainly expressed in the kidney, parathyroid gland, and choroid plexus, where it exists as a membrane-bound protein. $\alpha$-Klotho also can be found as a soluble protein in the blood, urine, and cerebrospinal fluid. Deficiency of $a$-Klotho results in VC, atherosclerosis, skin atrophy, and osteoporosis, a syndrome resembling human aging. Interestingly, these phenotypes of a-Klotho deficiency are similar to CKD subjects, suggesting that $\alpha$-Klotho is tightly related to the pathogenesis mechanisms of CKD [4]. Meanwhile, many researchers and our group have found that CKD is a state of severe a-Klotho deficiency, which is always along with progressive renal insufficiency and an extremely high incidence of CVD such as VC, cardiac hypertrophy, and uremic vasculopathy [5-8]. Conversely, exogenous supplement of $\alpha$-Klotho protein or genetic overexpression of $\alpha$-Klotho can remarkably attenuate renal dysfunction and decline morbidity and mortality of CVD complications [9], suggesting that $\alpha$-Klotho might be a predictor and a potential therapeutic target for renal and extra-renal outcomes in CKD. Based on the important relationship between $\alpha$-Klotho and CKD, we will review the physiopathological functions and potential applications of $\alpha$-Klotho in CKD and its complications below. Hereinafter the term Klotho is referred to as $\alpha$-Klotho.

\section{An Introduction of Klotho}

\section{Structure of Klotho Protein}

Klotho gene consists of 5 exons in both mice and humans. The Klotho protein is composed of a large extracellular domain and a short C-terminal intracellular region [10]. The extracellular domain consists of 2 repetitive sequences termed as $\mathrm{Kl} 1$ and $\mathrm{Kl} 2$. The encoded protein of full-length transcript can be cleaved by $a$-secretase metalloproteinase domain-containing proteins 10 and 17 (ADAM10 and ADAM17) as well as $\beta$-secretase $\beta$-site APP-cleaving enzyme 1 (BACE1). The cleaved Klotho is composed of $\mathrm{Kl} 1$ and $\mathrm{Kl} 2$, and both $\mathrm{Kl} 1$ and $\mathrm{Kl} 2$ fragments can release into circulation [11]. Secreted Klotho is synthesized by alternative transcription splicing. Soluble
Klotho includes cleaved Klotho and secreted Klotho. Membrane-bound Klotho exerts its functions mainly as co-receptor of fibroblast growth factor (FGF) family by increasing affinity of FGF receptors (FGFRs) to FGF [12]. Soluble Klotho acts as a hormone in the circulation to exert its role on the whole body.

Recently, 2 other homologous types of Klotho, $\beta$ - and $\gamma$-Klotho, were identified. $\beta$-Klotho contains $\mathrm{Kl} 1$ and $\mathrm{Kl} 2$ domains and is mainly expressed in liver, gastrointestinal tract, kidney, and adipose tissue. $\gamma$-Klotho contains only Kl1 domain and is expressed in adipose tissue, kidney, and skin [8]. We only focus on the role of $\alpha$-Klotho.

\section{Biological Functions of Klotho}

Soluble Klotho exerts its broad biological functions on targets, even several distal organs, as a pleiotropic endocrine/paracrine factor or a hormone, while membranebound Klotho always exerts its functions as co-receptors [13]. Of note, accumulating evidence suggest that soluble Klotho also can act as co-receptors for some special soluble ligands [14]. Thus, it is necessary to classify the different mechanisms underlying the Klotho functions.

\section{Membrane-Bound Klotho Acts as a Co-Receptor}

with FGF23

Membrane Klotho mainly forms co-receptors with FGFRs (FGFR1c, FGFR3c, and FGFR4) to promote the binding of FGF23 and FGFRs and regulate calcium and phosphorus metabolism. FGF23 is a bone-derived growth factor that belongs to FGF family [15]. Due to lack of a heparan sulfate-binding domain, FGF23 requires Klotho to convert the FGFR from canonical form into specific high-affinity form to function on target organs such as kidney and parathyroid glands. In this process, Klotho serves as a massive scaffold, tethering both FGFR and FGF23 to itself, enforcing proximity and augmenting binding affinity between these 2 molecules. The membrane Klotho-FGFRs-FGF23 complex could inhibit phosphate reabsorption by inhibiting NaPi-IIa in renal proximal tubule. At the same time, the complex can also downregulate the expression of renal $1 \alpha$-hydroxylase. As a result, the synthesis of 1,25-dihydroxyvitamin D3 (calcitriol) decreases, and the intestinal absorption of phosphate is reduced [16].

\section{Membrane-Bound Klotho Acts as a Competitor for} Binding Sites

FGF2 was found to be the founding member of the FGF family, and it was primarily identified as mitogens promoting proliferation of fibroblasts and vascular endo- 
thelial cells [17]. Unlike FGF23, FGF2 has heparin-binding domains, through which FGF2 tethers themselves to heparan sulfate in the extracellular matrices and functions primarily in an autocrine or paracrine manner [18]. Increased FGF2 and reduced Klotho have both been reported to be closely associated with renal fibrosis. Our group has demonstrated there was a competitive relationship between Klotho and FGF2, increase in FGF2 might weaken the function of Klotho, whereas the increase in Klotho would suppress the activity of FGF2 signaling pathway [19]. In addition, Klotho restrained FGF2-induced tubulo-epithelial plasticity and fibroblast proliferation and activation. The inhibitory effect of Klotho on the activity of FGF2 was likely due to its potent ability to compete with FGF2 binding to FGFR1, suggesting that there is a negative feedback loop exist between Klotho depletion and FGF2 activation [19].

Soluble Klotho Acts as a Co-Receptor for Soluble Ligands

Kusaba et al. [20] suggested that vascular endothelium in Klotho-deficient mice showed hyperpermeability with increased apoptosis and decreased vascular endothelialcadherin because of an increase in vascular endothelial growth factor (VEGF)-mediated internal calcium concentration $\mathrm{Ca}^{2+}$ influx and hyperactivation of $\mathrm{Ca}^{2+}$-dependent proteases. Nevertheless, Klotho supplement could directly strengthen the binding affinity between VEGF receptor 2 and endothelial transient receptor potential canonical $\mathrm{Ca}^{2+}$ channel 1 (TRPC1) to promote their co-internalization, the resulting regulating TRPC1mediated $\mathrm{Ca}^{2+}$ entry to maintain endothelial integrity.

Soluble Klotho Acts as an Enzyme

Previous studies showed that soluble Klotho possessed sialidase activity and regulated several ion channels via this activity. Removal of terminal sialic acids from $\mathrm{N}$-glycan chains of the epithelial $\mathrm{Ca}^{2+}$ channel TRPV5 and the renal $\mathrm{K}^{+}$channel renal outer medullary potassium channel 1 (ROMK1) by soluble Klotho exposes the underlying ligand for galectin-1. Binding to galectin-1 at the extracellular surface prevents internalization and leads to accumulation of the channels on the plasma membrane [21]. Soluble Klotho also downregulates transient receptor potential $\mathrm{Ca}^{2+}$ canonical isoform 6 (TRPC6)-mediated calcium signaling by binding to a2,3-sialyllactose moiety of gangliosides in lipid rafts [22]. Recent study has shown that soluble Klotho downregulated PI3K/Akt signaling via binding to ganglioside-enriched lipid [23]. These results suggest that Klotho regulates ion transport by mod- ifying sugar moieties of their transporters and affecting cell surface abundance via sialidase or $\beta$-glucuronidase activity [24].

Soluble Klotho Acts as a Decoy Receptor for Soluble Factors

Wnt proteins are a large family of highly conserved signal molecules that involved in process of nephron formation and renal development. Increasing evidence indicates that abnormal activation of $\mathrm{Wnt} / \beta$-catenin signaling pathways plays a crucial role in renal fibrosis. There is an inverse correlation between Klotho and canonical Wnt signaling. Soluble Klotho directly inhibits Wnt signaling by binding to several Wnt factors, such as Wnt1, Wnt4, and Wnt7a [25].

\section{Soluble Klotho Decreases Receptor Affinity for} Ligands

Soluble Klotho protein can directly bind to the type-II TGF- $\beta$ receptor (TGF $\beta$ R 2 ) and inhibit TGF- $\beta 1$ binding to cell surface receptors, thereby inhibiting TGF- $\beta 1$ signaling-induced epithelial-to-mesenchymal transition responses in cells. In addition to TGF- $\beta 1$ signaling, soluble Klotho has been shown to act as a circulating hormone that binds to a cell surface receptor and represses intracellular signals of insulin and insulin-like growth factor 1 (IGF1), an evolutionarily conserved mechanism for extending life span [26-28].

\section{Antioxidative Stress and Anti-inflammatory Effects of Klotho Protein}

Oxidative stress results from the imbalance between excessive oxidant free radicals and insufficient degradation of these prooxidants by anti-oxidants. CKD is a state of severe oxidative stress due to decreased antioxidants and excessive reactive oxygen species (ROS) generation [29]. Excessive ROS production in CKD state is involved in many kinds of CKD-associated complication [30]. On the contrary, controlling ROS is regarded as an effective way to prevent CKD-induced disease. Klotho is considered as an important antioxidative factor, and previous studies have found that there is a close connection between Klotho and ROS production. Klotho could inhibit insulin/IGF-1/PI3K signaling, then activating the FoxO forkhead transcription factors and inducing the expression of manganese superoxide dismutase to resist oxidative stress [31]. Interestingly, this antioxidative effect induced by Klotho also inhibits the production of mito- 
Table 1. The verified and potential mechanisms for cardiovascular protection of Klotho in CKD

\begin{tabular}{|c|c|c|c|}
\hline Animal model & $\begin{array}{l}\text { Outcome } \\
\text { of CVD }\end{array}$ & Mechanism & Reference \\
\hline EFmKL46 (Tg-Kl) mice & VC & Inhibition of Pi influx into VSMC & Hu et al., $2011[48]$ \\
\hline Klotho $^{-1-}$ mice + rapamycin & $\mathrm{VC}$ & Inhibition of mammalian target of rapamycin (mTOR) & Zhao et al., 2015 [110] \\
\hline $\mathrm{NaPi} 2 \mathrm{a}^{-/-} / \mathrm{Klotho}^{-/-}$mice & $\mathrm{VC}$ & Regulating the phosphate associated co-transporter & Ohnishi et al., 2009a [51] \\
\hline Cyp27b1 $1^{-/-} /$Klotho $^{-/-}$mice & $\mathrm{VC}$ & Reducing vitamin D levels through inactivation of Cyp27b1 activity & Ohnishi et al., 2009b [52] \\
\hline Uremic mice + Klotho i.p. & LVH & $\begin{array}{l}\text { Blocking Nox } 2 / \text { Nox } 4 \text {-derived ROS production and inhibiting p } 38 \text { and ERK1/2 } \\
\text { signaling pathways }\end{array}$ & Yang et al., 2015 [6] \\
\hline Diabetic mice + Klotho i.p. & LVH & Suppressing cardiac inflammatory cytokines and oxidative stress & Guo et al., 2018 [68] \\
\hline Uremic rats + rKlotho & LVH & Increasing myocardial fibroblast growth factor 21 expression & Suassuna et al., 2020 [73] \\
\hline $\begin{array}{l}\text { Mice received subcutaneous } \\
\text { injections of Ang II + Klotho }\end{array}$ & LVH & Modifying the TGF- $\beta 1-\mathrm{miR}-132$ axis & Ding et al., 2019 [67] \\
\hline Klotho $^{-1-}$ mice & AS & Regulating TRPC-1-mediated $\mathrm{Ca}^{2+}$ entry & Kusaba et al., 2016 [20] \\
\hline OLETF rats + Ad-Klotho & AS & Increasing nitric oxide production & Saito et al., 2000 [111] \\
\hline DKD mice + pcMV-Klotho & AS & $\begin{array}{l}\text { Inhibiting macrophage } \mathrm{M} 1 \text { polarization and inducing macrophage } \mathrm{M} 2 \\
\text { polarization }\end{array}$ & Jia et al., 2019 [95] \\
\hline CKD mice + Klotho i.p. & AS & Inhibiting platelet hyperactivity & Yang et al., 2017 [7] \\
\hline
\end{tabular}

chondrial-derived ROS and protects mitochondrial dysfunction [32]. In addition, it has been reported that increased endogenous ROS production in Klotho-deficient mice and reduced ROS production in Klotho overexpression are closely related to the regulation of ASK1signalosome-p38 MAPK signal pathway [33].

$\mathrm{CKD}$ is regarded as a typical example of inflammatory disease and premature ageing. Plenty of pro-inflammatory factors accumulated during CKD progression. These inflammatory factors play an important role in CKD-induced complication. There are multiple studies that have shown a bidirectional relationship between Klotho and inflammation. On the one hand, inflammation can decrease the expression of Klotho through transcriptional and epigenetic mechanisms [34]. On the other hand, Klotho is reported to have powerful anti-inflammatory effect. Our group found that Klotho could inhibit indoxyl sulfate (IS)-activated RIG-I/NF- $\kappa B$ and the production of inflammatory factors including IL- 6 and TNF- $\alpha$ in cultured monocytes. Interestingly, higher serum levels of IL- 6 and TNF- $\alpha$ were observed in Klotho heterozygous mice with $\mathrm{CKD}$ than that in single $\mathrm{CKD}$ group. Conversely, Klotho treatment can dramatically inhibit inflammation in monocytes. Previous report has shown that sup- plementation or overexpression of Klotho in vitro could suppress NF- $\kappa \mathrm{B}[35]$ and RIG-I-mediated inflammation [36], which is consistent with our results. Moreover, it has been reported that post-treatment with recombinant Klotho could suppress the inflammatory responses in aging endotoxemic mice through modulating levels of heat shock protein 70 [37]. These findings suggest that Klotho has a powerful antioxidative stress and anti-inflammatory ability.

\section{The Role of Klotho in CKD-Induced CVD}

Physiologically, kidney is the major organ to maintain soluble Klotho homeostasis. Yet in CKD, a large number of renal tubular epithelial cells are injured and replaced by extracellular matrix, and the rest cannot produce and release enough soluble Klotho into the circulation. Therefore, CKD is proposed as a state of soluble Klotho deficiency [38]. Even in the younger CKD population such as children, serum level of Klotho was still lower than in healthy subjects. As CKD and Klotho-deficient mice have an extremely high incidence of CVD [4], the morbidity and mortality of CVD can be repressed by Klotho gene 
Fig. 1. The role of Klotho in CKD-associated CVD. CKD is a public health epidemic. CVD, including uremic cardiomyopathy, vascular calcification, and atherosclerosis, was found in CKD subjects. Klotho deficiency, which results from renal insufficiency, is always associated with poor outcomes in CKD. By contrast, prevention of Klotho decline by inhibiting oxidative stress and inflammation as well as rectifying mineral disturbance can dramatically mitigate cardiac dysfunction, prevent vascular calcification, and retard the progression of CKD-accelerated atherosclerosis. AS, atherosclerosis; CVD, cardiovascular disease; LVH, left ventricular hypertrophy; VC, vascular calcification.

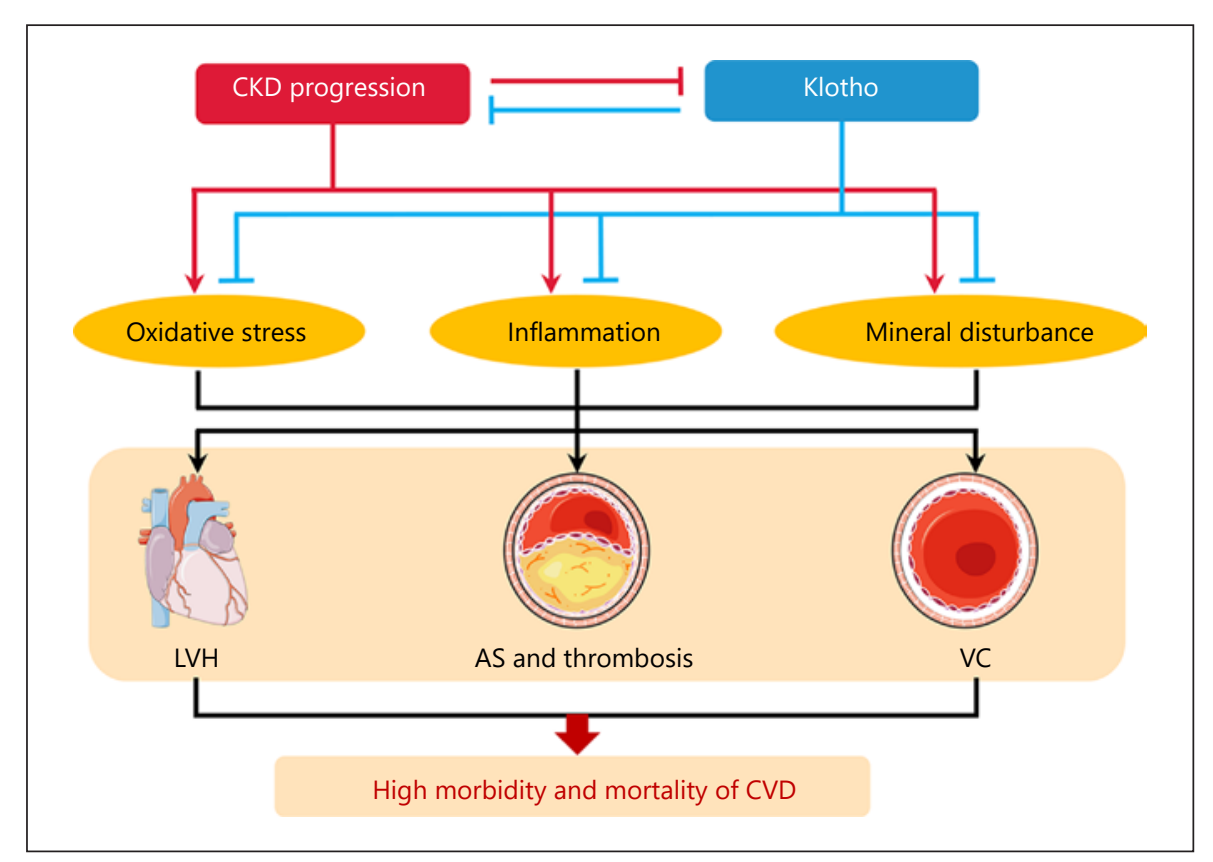

overexpression or protein supplementation in those animal models $[39,40]$. We summarized the in vivo studies directly related to the protective effects of Klotho (shown in Table 1). These phenomena suggest that loss of Klotho is likely to be directly pathogenic and probably a prerequisite for CKD-associated CVD (shown in Fig. 1).

\section{The Role of Klotho in VC}

$\mathrm{VC}$ is one of the most potent risk factors of cardiovascular mortality and morbidity in CKD patients, and CKD is often related to vascular media calcification. Even in the early stage of CKD, the rate of $\mathrm{VC}$ increases significantly [41]. VC is mainly related to the transdifferentiation of vascular smooth muscle cells (VSMCs) into osteogeniclike cells. CKD-induced mineral bone disorder syndrome is a potent contributor to VC [42]. Plenty of evidence revealed that disturbance of serum phosphate and calcium can directly lead to VC $[43,44]$. Previous study has found that hyperphosphatemia was regarded as a main inducer for the onset and progression of VC. Under the influence of CKD, both hyperphosphatemia and VC represent an extremely higher incidence than the health population. However, CKD patients with normal serum phosphate levels still showed severe VC, suggesting that several factors other than hyperphosphatemia can lead to VC [45]. Recently, the role of Klotho is considered increasingly important in CKD-induced VC. It has been reported that either in CKD patients or peritoneal dialysis patients, low serum levels of soluble Klotho are independently related to VC $[46,47]$. In CKD mice, Klotho deficiency induces a higher serum phosphate level and more severe VC than that in CKD mice. On the contrary, overexpression or supplementation of Klotho can reduce serum phosphate level, improve renal function, and protect against VC [48]. In vitro, using Klotho siRNA pretreatment accelerates CKD-induced VSMCs transformation to a calcifying phenotype, which is characterized by the loss of inhibitory factors, such as MGP (matrix Gla protein) and OPN (osteopontin), and upregulation of Runx2 (runt-related transcription factor 2) [49]. These results suggest that the deficiency of Klotho is not only a marker CKD, but is also an important risk factor that contributes to the onset and progression of VC in CKD.

The mechanism of Klotho deficiency aggravating CKD-induced VC is closely related to the homeostasis of phosphorus metabolism. Klotho could regulate phosphate metabolism by enhancing phosphaturia and preserving glomerular filtration, as complete Klotho deficiency induces high serum phosphate levels [48]. Previous studies indicated that restricted dietary phosphate of Klotho-deficient mice might increase renal expression of Klotho and partially rescued the ectopic calcification [50, 51]. Besides, it is reported that $\mathrm{NaPi} 2 \mathrm{a}^{-/-} \mathrm{Klotho}^{-/-}$mice showed lower phosphate levels and reduced $\mathrm{VC}$, while the features of VC reestablished as long as the mice were given the same mice high-phosphate diets [51]. It is therefore conceivable that in the setting of Klotho deficiency, elevated phosphate is indispensable to VC [52]. In fact, de- 
spite lower phosphate diets, serum phosphate eventually increased to the point of inducing VC. This may be mediated by increased activation of $\mathrm{NaPi} 2 \mathrm{a}$ induced by elevated FGF23 and lack of compensatory action resulting from Klotho deficiency. In contrast to low-phosphate diets, low-calcium diets could effectively restrain the progression of calcification [53]. These effects suggest that in the setting of Klotho deficiency, calcium may be another ratelimiting factor for VC pathophysiology.

Supplementation or overexpression of Klotho could play a protective role in CKD-induced VC. Hum et al. [54] reported that injection of recombinant Klotho downregulated the expression of renal sodium-phosphate cotransporter Npt2a, which reduced hyperphosphatemia and eventually rescued the CKD-associated VC. Moreover, CKD mice with overexpression of Klotho showed a less severe VC than wild-type CKD mice. In vitro, it has been found that overexpression of Klotho directly inhibited phosphate influx into VSMC, which in turn suppressed the differentiation of VSMC and rescued the VC [48]. These results demonstrate that Klotho is an endogenous inhibitor of VC [49]. The mechanism of protective role of Klotho in VC is related to the ability to inhibit the uptake of phosphate through phosphate sodium-dependent co-transporter in VSMCs [55]. In addition, we have investigated that local expression of Klotho in artery had ability to inhibit VC by regulating phosphate uptake. And the expression of Klotho is decreased with the peroxisome proliferator-activated receptor-gamma (PPAR- $\gamma$ ) reduction in VSMCs after high phosphate treatment [56]. Another mechanism is that Klotho has anti-oxidative and anti-apoptotic effects in VSMCs to decease VC. Oxidative stress and apoptosis are important factors to induce VC in CKD [45, 57]. Wang et al. [58] found that Klotho gene transfer significantly decreased superoxide production by decreasing the expression of Nox2 in VSMCs in protein level through inhibiting cAMP-PKA pathway. It has also been reported that exogenous Klotho ameliorated the development of VC and ROS generation induced by salusin- $\beta$ [59]. Besides, it has also been found that Klotho can protect AngII-induced VSMCs apoptosis. Finally, Klotho can be inducible by other substances and then acts as an induced protective factor to inhibit VC. Upregulation of Klotho expression by inhibiting mTOR signaling via oral rapamycin ameliorates $\mathrm{VC}$ and protects against vascular disease in CKD [60]. Another study showed that intermedin 1-53 attenuates VC in rats with CKD by upregulating membrane-bound Klotho expression in the vessel wall [61]. All of these suggested that Klotho could be a hopeful therapeutic target for CKD-induced VC and whether maintaining or increasing the Klotho level could improve VC in CKD patients is needed to be proven in future research.

\section{The Role of Klotho in Left Ventricular Hypertrophy}

The epidemiological studies show that the progressively declined renal function accompanied by a high incidence of left ventricular hypertrophy (LVH), which accounts for nearly 40 and $74 \%$ in patients with early stage of CKD and ESRD, respectively. LVH is the pathological basis of heart failure or ventricular arrhythmias, which is regarded as an important independent risk factor in patients with CKD [62]. Previous studies indicated that pressure and volume overload were the major contributors to $\mathrm{LVH}$ in CKD. However, LVH still develops even after mitigating these 2 factors [63]. Recent data prefer to pay more attention on the role of Klotho in CKD-induced LVH. It has been reported that maintenance hemodialysis patients with lower circulating sKlotho levels were more often associated with larger interventricular septal thickness and greater ratios of interventricular septal thickness and posterior wall thickness, which are typical indicators of LVH in patients [64]. Consistently, in a survey of 86 patients with $\mathrm{CKD}$, we found that increased left ventricular mass was more apparent in patients with low Klotho level [6]. In mice, it has been reported that Klotho-deficient CKD mice showed worsening LVH, while LVH in Klotho-overexpressing CKD mice was relieved [65]. These suggested that there was an independent association between Klotho and CKD-induced LVH, and Klotho could be a potential risk factor of uremic cardiomyopathy. Therefore, discussing the role of Klotho in this pathological process is helpful to understand the underlying mechanism of CKD-induced LVH and find effective treatment.

It has been indicated that administered injections or genetically active expression of Klotho could apparently attenuate cardiac remodeling, manifested as reduced ventricular wall thickness, left ventricular internal diameter at end-diastole (LVIDD), and posterior wall thickness in mice $[6,65]$. The cardioprotective role of Klotho in CKD is mainly related to its robust antioxidative stress effect. Recent data showed that Klotho could protect against cardiomyocyte hypertrophy induced by isoproterenol [66], Ang II [67], or hyperglycemia [68] by suppressing ROSstimulated signaling pathways both in vitro and in vivo experiments. We proved that Klotho protects IS-induced LVH by inhibiting oxidative stress signaling pathway in vivo and in vitro. In detail, we found that IS induced cardiomyocyte hypertrophy by activating oxidative stress and its downstream signaling pathways p38 and ERK1/2 
and Klotho showed powerful antioxidative ability to ameliorate the pathological changes [6].

Second, Klotho can act as circulating co-receptor that can bind circulating FGF23 and FGFRs on the cell surface, which makes it possible for Klotho to regulate FGF23-mediated signaling [69]. As a novel cardiovascular uremic toxin, FGF23 directly participates in the development of LVH in CKD. Evidence showed that FGF23 directly targeted cardiomyocytes by activating FGFR4/ PLC $\gamma /$ calcineurin/NFAT signaling pathway and induced cardiac hypertrophy [70]. Interestingly, it is suggested that Klotho can regulate myocardial FGF23 effects by blocking the binding of circulating FGF23 to FGFR4 on cardiomyocyte membrane [71, 72]. Besides, Klotho could also play a role of co-receptor for FGFR1c to redirect the action of FGF23-induced cardioprotective pathway. One of the possible protective pathways is to induce the expression of FGF21 [73]. In addition, Klotho can ameliorate cardiac hypertrophy through inhibiting TRPC6 currents by blocking phosphoinositide-3-kinase-dependent exocytosis of TRPC6 channels in uremic hearts [74]. Further study made it clear that this effect of Klotho-inhibited TRPC6 was independent of FGF23 $[22,75]$.

Finally, Klotho can directly prevent cardiac remodeling and fibrosis to protect the heart. Cardiac remodeling is characterized by cardiomyocyte hypertrophy and fibrosis and has been regarded as an important risk factor for the development of heart failure in CKD $[76,77]$. Recent study showed that Klotho protein can protect Ang II-induced cardiac remodeling through regulating TGF$\beta$-miR-132 axis. Liu et al. [78] reported that Klotho is expressed in human atrial appendage tissues. Further, Klotho knockdown or overexpression in primary human cardiomyocytes aggravates or mitigates TGF- $\beta 1$-induced cardiac fibrosis and activation of the Wnt signaling. These results suggest that endogenous Klotho in cardiomyocytes plays an important role in CKD-induced cardiac fibrosis and subsequent activation of Wnt signaling pathway. On the contrary, upregulation of endogenous Klotho can inhibit activation of Wnt signaling pathway induced by $\mathrm{CKD}$, which may provide a new strategy for the treatment of myocardial fibrosis in CKD patients. All of these evidence show that Klotho participates in the pathogenesis of CKD-associated LVH and treatment with Klotho is a potential therapy for CKD-induced $\mathrm{LVH}$.

\section{The Role of Klotho in CKD-Accelerated}

Atherosclerosis

As a chronic inflammatory disease with complex etiology and the most potent pathological basis of multiple cardiovascular events, atherosclerosis is seriously harmful to human health. The major clinical outcomes of atherosclerosis, such as myocardial infarction or stoke, are not a result of gradual narrowing of lumen but rather the cause of thrombotic events [79]. Of note, atherosclerosis is the leading cause of cardiovascular morbidity and mortality in CKD patients and its progression is often accelerated [80]. Over the past decades, considerable efforts have been devoted to investigate the risk factors as well as their underlying mechanisms that accelerated development and progression of atherosclerosis in CKD.

As an early predictor of atherosclerosis, lower levels of Klotho are positively correlated with lower values of flowmediated dilation, larger values of epicardial fat thickness and carotid artery intima-media thickness, all of which are the admitted predictors of atherosclerosis [81]. Moreover, lower Klotho levels coexist with higher inflammatory status in patients with atherosclerosis [82]. In the hemodialysis population, Klotho acts as an atherosclerosis predictor as well. Increasing numbers of studies showed that Klotho deficiency can aggravate the progress of atherosclerosis in CKD, while supplement of Klotho played a protective role in this process [83]. Therefore, it is significant to focus on the effects of Klotho in this pathological process, and here, we will discuss the role of Klotho in the pathogenesis of atherosclerosis in CKD to provide clues for future research.

\section{Protection against Endothelial Dysfunction}

Endothelial dysfunction plays a vital role in the onset and progression of CKD-induced atherosclerosis [84]. Previous study has found that endothelial dysfunction was a common feature of arteries in Klotho-deficient mice [20]. Conversely, many endothelial protective factors such as reduced systolic blood pressure, coronary perivascular fibrosis, and medial hypertrophy were observed in Klotho transgenic mice [85]. It has been reported that membrane-form Klotho enhanced manganese superoxide dismutase expression by approximately twofold, partially via activation of the cAMP/PKA-dependent pathway and increase of nitric oxide production in human umbilical vein endothelial cells [86]. This finding provides new insights into the mechanisms of Klotho action and support the therapeutic potential of membraneform Klotho to regulate endothelial function. In addition, Cui et al. [87] suggested that Klotho protected $\mathrm{H}_{2} \mathrm{O}_{2}$-induced oxidative injury by activating the PI3K/AKT pathway in endothelial cells. Similarly, Klotho can attenuate ox-LDL-induced oxidative stress in human umbilical vein endothelial cells through upregulating oxidative 
scavengers (superoxide dismutase and $\mathrm{NO}$ ) via activating the PI3K/Akt/eNOS pathway and suppressing LOX-1 expression [88]. Moreover, we demonstrated that Klotho could attenuate the effects of uremic toxin-induced activation of $\mathrm{p} 38 \mathrm{MAPK}, \mathrm{NF}-\mathrm{\kappa B}$, and ROS production and protect endothelial cells from the injury [5]. Also, Klotho had an ability to inhibit endothelial senescence via mitogen-activated protein kinase kinase and extracellular signal-regulated kinase pathways [89]. Besides, Liu et al. [90] demonstrated that Klotho participated in microRNA335-5p-induced endothelial senescence. These evidence suggested the protective effects of Klotho in protecting endothelial dysfunction.

\section{Protection against VSMC Injuries}

In the early stage of atherosclerosis, the proliferation and migration of VSMCs dramatically promote the progression of atherosclerosis [91]. Interestingly, Klotho can inhibit proliferation and migration of Ang II-induced VSMCs by regulating NF- $\mathrm{BB} / \mathrm{p} 65 / \mathrm{Akt} / \mathrm{ERK}$ signaling pathways. Klotho also inhibited the expression of proliferation phenotype marker protein, PCNA, and increased the expression of contractile phenotype marker proteins, suggesting that Klotho plays a major role in Ang II-induced phenotype modulation of VSMCs [92]. Moreover, Klotho was proposed to protect VSMCs by inhibiting oxidative stress. It is suggested that VSMCs with overexpression of Klotho significantly reduced the expression of Nox2 and ROS production through regulating cAMP/ PKA pathways [58]. In addition, it has been shown that Klotho supplement can enhance the expression of oxygenase-1 (HO-1) and peroxiredoxin-1 (Prdx-1), which are powerful antioxidant enzymes to protect against VSMC dysfunction. During this process, Nrf2 activation was found after the supplement of Klotho, and silencing of Nrf2 attenuated the induction of HO- 1 and Prx-1 induced by Klotho [93]. These results suggested that Nrf2mediated antioxidant roles of Klotho in VSMCs.

\section{Inhibiting Macrophage/Monocyte-Associated}

Inflammation and Phenotypic Transition

Recently, we found that lack of Klotho aggravates systemic inflammation in CKD, while supplement of Klotho might mitigate this process by decreasing the expression of inflammatory factors or related signaling pathways [94], which hints that Klotho could prevent atherosclerosis progression through inhibiting monocyte-associated inflammation. In addition, Klotho participated human serum albumin and MiR-199a-5p induced macrophage M1 polarization, which is a pro-inflammatory pheno- type. Further, Klotho induces macrophage M2 polarization through the Toll-like receptor 4 pathway both in vivo and in vitro experiments [95]. These findings hint that Klotho might be involved in the phenotype modulation of macrophage and the progression of atherosclerosis. Finally, researchers suggested that Klotho can inhibit atherosclerosis by lowering lipid. Pretreatment of Klotho protein before incubating with phorbol myristate acetate and ox-LDL can significantly decrease total cholesterol level and suppressing transition of THP-1 macrophages to foam cells. Furthermore, Klotho-induced upregulation of reverse cholesterol transport capacity promotes cholesterol efflux and reduces lipid accumulation by suppressing the Wnt/ $\beta$-catenin pathway in foam cells [96].

Inhibiting Platelet Hyperactivity

Activated platelets are present in the circulating blood of atherosclerotic individuals throughout the atherosclerotic process, which play an important part in the atherosclerotic lesions. Activated platelets prefer to bind leukocytes and form platelet-leukocyte aggregates [97]. In both in vivo and in vitro studies, it had been proved that aggregation of the circulating activated platelets and platelet-leukocyte promoted the development of atherosclerosis [98]. In both CKD and IS-treated mice, we found that the expression of P-selectin and the circulating level of PMPs were remarkably increased, while activated GPIIb/IIIa induced by collagen and thrombin was more obvious and a high tendency of thrombus formation [7]. These suggested that IS plays an important role in hyperactivation of platelets and the formation of thrombosis in CKD. Furthermore, we found that the activation of platelets induced by IS in CKD state is caused by the activation of ROS/p38 MAPK signaling. As expected, Klotho treatment markedly ameliorated IS-induced platelet hyperactivity through inhibiting IS-induced activation of ROS/p38 MAPK signaling and reduced the formation of thrombosis both in vitro and in vivo [7]. Thus, we concluded that Klotho plays a key protective role in CKD-induced atherosclerosis and thrombosis through inhibiting IS-induced platelet hyperactivity.

\section{The Prospect of Clinical Application of Klotho in \\ CKD-Associated CVD}

We have discussed the definite protective role and the underlying mechanisms of Klotho in CKD-associated CVD. Therefore, the prospect of clinical application of Klotho is of great concern. CKD-associated CVD is the main cause of death in patients with $\mathrm{CKD}$, which occurs 
in the early stage of CKD and progresses rapidly. It is very difficult to make an early and accurate diagnosis. And there are no biomarkers that are able to be measured easily, specially, and sensitively, in correlation with CKDassociated CVD complications [99, 100]. Recently, several clinical studies found that there was an excellent correlation between Klotho and cardiovascular events or mortality in CKD patients. In dialysis patients, Marçais et al. [101] reported that conservation of serum Klotho above $280 \mathrm{ng} / \mathrm{L}$ is associated with a better 2-year cardiovascular protection. This is consistent with a recent prospective study - lower serum level of Klotho is associated with cardiovascular events in hemodialysis patients [102]. In CKD patients without dialysis, we demonstrated that lower serum Klotho levels are independently associated with overall mortality and CVD events [103]. Collectively, it is clear that Klotho is an independent predictor of CVD complication and cardiovascular events in CKD patients. However, large multicenter clinical cohort studies are required to determine the cutoff value of Klotho for predicting the onset of CVD complication in CKD patients. It is definitely helpful for clinicians to judge the patient's cardiovascular-related condition and prognosis.

In addition, the clinical transformation studies of Klotho should be carried out in a planned way, and it will make it clear that whether the increase of serum Klotho exerts protective effects on cardiovascular events in patients. Potential drugs to increase the serum Klotho level include PPAR- $\gamma$ agonists, angiotensin II-type I receptor antagonists, vitamin $\mathrm{D}$ active derivatives, intermedin, and dihydromyricetin, all of which had been proved to upregulate the expression of Klotho in vivo and/or in vitro $[38,61,104-106]$. Furthermore, direct supplementation of soluble Klotho protein is also a hopeful treatment. However, potential side effects of increasing serum Klotho level should also be taken into account. Some researchers have reported the potential side effects of Klotho in inducing insulin resistance and participating in obesity and obesity-associated complications [107, 108]. Furthermore, mechanism of Klotho mediated-insulin resistance is through prevention of GLUT4 translocation and interfering with phosphorylation of Akt, GSK $3 \beta$, and PFKf $3 \beta$ intracellular signaling mediators by insulin [108]. However, this point of view is controversial, as Lorenzi et al. [109] provided evidence against a direct role of Klotho in insulin resistance in HEK293, L6, and HepG2 cells. Therefore, more studies are needed to clearly determine the potential side effects of Klotho protein.

Klotho in CKD-Associated CVD

\section{Conclusion}

The identification of Klotho helps us to understand more about how Klotho functions as a circulating hormone or local autocrine/paracrine factor. Klotho exerts pleiotropic functions in body. In future study, the crystal structure, functional pattern, as well as the regulatory mechanisms of Klotho are needed to be discovered. What is more, it is also unclear whether there is a receptor on the cell membranes that directly binds to Klotho. A large number of basic and clinical studies have shown that Klotho is an excellent predictor of CKD-related cardiovascular complications. The in-depth researches of the relation between Klotho and CVD complications in CKD as well as its potential applications are the potential therapeutic strategies for CKD-associated CVD in the future.

\section{Conflict of Interest Statement}

All the authors declared no competing interests.

\section{Funding Sources}

This study was supported by research grants from the Natural Science Foundation of China (No. 81873605, 81700379), the Young Elite Scientist Sponsorship Program by CAST (No. 2018QNRC001), Personal Training Program for Clinical Medicine Research of Army Medical University (No. 2018XLC1007), and Frontier Specific Projects of Xinqiao Hospital (No. 2018YQYLY004).

\section{Author Contributions}

J.Z. decided on the topics. X.B. and K.Y. wrote the manuscript and prepared the figure and the table. B.Z. provided suggestions for the revision of the manuscript.

\section{References}

1 Zoccali C, Vanholder R, Massy ZA, Ortiz A, Sarafidis P, Dekker FW, et al. The systemic nature of CKD. Nat Rev Nephrol. 2017 Jun; 13(6):344-58.

2 Sadeghi-Alavijeh O, Tadayyon M, Caplin B. Chronic kidney disease-associated cardiovascular disease: scope and limitations of animal models. Cardiovasc Endocrinol. 2017;6(4):120-7.

3 Kuro-o M, Matsumura Y, Aizawa H, Kawaguchi $\mathrm{H}$, Suga T, Utsugi T, et al. Mutation of the mouse klotho gene leads to a syndrome resembling ageing. Nature. 1997 Nov 6;390 (6655):45-51.

4 Zou D, Wu W, He Y, Ma S, Gao J. The role of klotho in chronic kidney disease. BMC Nephrol. 2018 Oct 22;19:285. 
5 Yang K, Nie L, Huang Y, Zhang J, Xiao T, Guan X, et al. Amelioration of uremic toxin indoxyl sulfate-induced endothelial cell dysfunction by Klotho protein. Toxicol Lett. 2012 Nov 30;215(2):77-83.

6 Yang K, Wang C, Nie L, Zhao X, Gu J, Guan $\mathrm{X}$, et al. Klotho protects against indoxyl sulphate-induced myocardial hypertrophy. J Am Soc Nephrol. 2015 Oct;26(10):2434-46.

7 Yang K, Du C, Wang X, Li F, Xu Y, Wang S, et al. Indoxyl sulfate induces platelet hyperactivity and contributes to chronic kidney disease-associated thrombosis in mice. Blood. 2017 May 11;129(19):2667-79.

8 Kuro-o M. The Klotho proteins in health and disease. Nat Rev Nephrol. 2019 Jan;15(1):2744.

9 Neyra JA, Hu MC. Potential application of klotho in human chronic kidney disease. Bone. 2017 Jul;100:41-9.

10 Olejnik A, Franczak A, Krzywonos-Zawadzka A, Kałużna-Oleksy M, Bil-Lula I. The biological role of Klotho protein in the development of cardiovascular diseases. Biomed Res Int. 2018 Dec 24;2018:5171945.

11 Lim K, Halim A, Lu T-S, Ashworth A, Chong I. Klotho: a major shareholder in vascular aging enterprises. Int J Mol Sci. 2019 Sep 19;20: 4637.

12 Muñoz-Castañeda JR, Rodelo-Haad C, Pendon-Ruiz de Mier MV, Martin-Malo A, Santamaria R, Rodriguez M. Klotho/FGF23 and Wnt signaling as important players in the comorbidities associated with chronic kidney disease. Toxins. 2020 Mar 16;12:185.

13 Kuro-O M. Molecular mechanisms underlying accelerated aging by defects in the FGF23klotho system. Int J Nephrol. 2018;2018: 9679841.

14 Dalton GD, Xie J, An SW, Huang CL. New insights into the mechanism of action of soluble Klotho. Front Endocrinol. 2017;8:323-3.

15 Schumacher D, Schuh A. Cardiac FGF23: a new player in myocardial infarction. Discoveries. 2019 Sep 30;7:e97.

16 Richter B, Faul C. FGF23 actions on target tissues-with and without klotho. Front Endocrinol. 2018;9:189.

17 Kuro-O M. Klotho and endocrine fibroblast growth factors: markers of chronic kidney disease progression and cardiovascular complications? Nephrol Dial Transpl. 2019 Jan 1; 34:15-21.

18 Schlessinger J, Plotnikov AN, Ibrahimi OA, Eliseenkova AV, Yeh BK, Yayon A, et al. Crystal structure of a ternary FGF-FGFR-heparin complex reveals a dual role for heparin in FGFR binding and dimerization. Mol Cell. 2000 Sep;6(3):743-50.

19 Guan X, Nie L, He T, Yang K, Xiao T, Wang $\mathrm{S}$, et al. Klotho suppresses renal tubulo-interstitial fibrosis by controlling basic fibroblast growth factor-2 signalling. J Pathol. 2014 Dec; 234(4):560-72.

20 Kusaba T, Okigaki M, Matui A, Murakami M, Ishikawa K, Kimura T, et al. Klotho is associated with VEGF receptor-2 and the transient receptor potential canonical-1 $\mathrm{Ca}^{2+}$ channel to maintain endothelial integrity. Proc Natl Acad Sci U S A. 2010 Nov 9;107(45):1930813.

21 Cha SK, Hu MC, Kurosu H, Kuro-o M, Moe $\mathrm{O}$, Huang CL. Regulation of renal outer medullary potassium channel and renal $\mathrm{K}(+)$ excretion by Klotho. Mol Pharmacol. 2009 Jul; 76(1):38-46.

22 Wright JD, An S-W, Xie J, Lim C, Huang C-L. Soluble klotho regulates TRPC6 calcium signaling lipid rafts, independent of the FGFRFGF23 pathway. FASEB J. 2019 Aug;33(8): 9182-93.

23 Dalton G, An SW, Al-Juboori SI, Nischan N, Yoon J, Dobrinskikh E, et al. Soluble klotho binds monosialoganglioside to regulate membrane microdomains and growth factor signaling. Proc Natl Acad Sci U S A. 2017 Jan 24; 114(4):752-7.

24 Huang C-L. Regulation of ion channels by secreted Klotho: mechanisms and implications. Kidney Int. 2012;77(10):855-60.

25 Satoh M, Nagasu H, Morita Y, Yamaguchi TP, Kanwar YS, Kashihara N. Klotho protects against mouse renal fibrosis by inhibiting Wnt signaling. Am J Physiol Renal Physiol. 2012 Dec 15;303(12):F1641-51.

26 Kurosu H, Yamamoto M, Clark JD, Pastor JV, Nandi A, Gurnani P, et al. Suppression of aging in mice by the hormone Klotho. Science. 2005 Sep 16;309(5742):1829-33.

27 Wolf I, Levanon-Cohen S, Bose S, Ligumsky H, Sredni B, Kanety H, et al. Klotho: a tumor suppressor and a modulator of the IGF-1 and FGF pathways in human breast cancer. Oncogene. 2008 Nov 27;27(56):7094-105.

28 Doi S, Zou Y, Togao O, Pastor JV, John GB, Wang $L$, et al. Klotho inhibits transforming growth factor-betal (TGF-beta1) signaling and suppresses renal fibrosis and cancer metastasis in mice. J Biol Chem. 2011 Mar 11; 286(10):8655-65.

29 Ravarotto V, Simioni F, Pagnin E, Davis PA, Calò LA. Oxidative stress: chronic kidney disease: cardiovascular disease: a vicious circle. Life Sci. 2018 Oct 1;210:125-31.

30 Huang $\mathrm{M}$, Zheng L, Xu H, Tang D, Lin L, Zhang J, et al. Oxidative stress contributes to vascular calcification in patients with chronic kidney disease. J Mol Cell Cardiol. 2020 Jan; 138:256-68.

31 Yamamoto M, Clark JD, Pastor JV, Gurnani P, Nandi A, Kurosu H, et al. Regulation of oxidative stress by the anti-aging hormone klotho. J Biol Chem. 2005 Nov 11;280(45): 38029-34.

32 Lim SW, Jin L, Luo K, Jin J, Shin YJ, Hong SY, et al. Klotho enhances FoxO3-mediated manganese superoxide dismutase expression by negatively regulating PI3K/AKT pathway during tacrolimus-induced oxidative stress. Cell Death Dis. 2017 Aug 3;8(8):e2972.

33 Hsieh CC, Kuro-o M, Rosenblatt KP, Brobey $\mathrm{R}$, Papaconstantinou J. The ASK1-Signalosome regulates p38 MAPK activity in response to levels of endogenous oxidative stress in the Klotho mouse models of aging. Aging (Albany NY). 2010 Sep;2(9):597-611.

34 Ruiz-Andres O, Sanchez-Niño MD, Moreno JA, Ruiz-Ortega M, Ramos AM, Sanz AB, et al. Downregulation of kidney protective factors by inflammation: role of transcription factors and epigenetic mechanisms. Am J Physiol Renal Physiol. 2016 Dec 1;311(6): F1329-40.

35 Jin M, Lv P, Chen G, Wang P, Zuo Z, Ren L, et al. Klotho ameliorates cyclosporine A-induced nephropathy via PDLIM2/NF-kB p65 signaling pathway. Biochem Biophys Res Commun. 2017 Apr 29;486(2):451-7.

36 Liu F, Wu S, Ren H, Gu J. Klotho suppresses RIG-I-mediated senescence-associated inflammation. Nat Cell Biol. 2011 Mar;13(3): 254-62.

37 Hui H, Zhai Y, Ao L, Cleveland JC, Liu H, Fullerton DA, et al. Klotho suppresses the inflammatory responses and ameliorates cardiac dysfunction in aging endotoxemic mice. Oncotarget. $2017 \mathrm{Feb}$ 28;8(9):15663-76.

38 Liu Y, Bi X, Xiong J, Han W, Xiao T, Xu X, et al. MicroRNA-34a promotes renal fibrosis by downregulation of klotho in tubular epithelial cells. Mol Ther. 2019 May 8;27(5):1051-65.

39 Donate-Correa J, Martín-Núñez E, MoraFernández C, Muros-de-Fuentes M, PérezDelgado N, Navarro-González JF. Klotho in cardiovascular disease: current and future perspectives. World J Biol Chem. 2015 Nov 26;6(4):351-7.

40 Takenaka T, Kobori H, Inoue T, Miyazaki T, Suzuki H, Nishiyama A, et al. Klotho supplementation ameliorates blood pressure and renal function in DBA/2-pcy mice, a model of polycystic kidney disease. Am J Physiol Renal Physiol. 2020 Mar 1;318(3):F557-64.

41 Dai L, Qureshi AR, Witasp A, Lindholm B, Stenvinkel P. Early vascular ageing and cellular senescence in chronic kidney disease. Comput Struct Biotechnol J. 2019;17:721-9.

42 Ray M, Jovanovich A. Mineral bone abnormalities and vascular calcifications. Adv Chronic Kidney Dis. 2019 Nov;26:409-16.

43 Ritter CS, Slatopolsky E. Phosphate toxicity in CKD: the killer among us. Clin J Am Soc Nephrol. 2016 Jun 6;11(6):1088-100.

44 Yamada S, Giachelli CM. Vascular calcification in CKD-MBD: roles for phosphate, FGF23, and Klotho. Bone. 2017 Jul;100:8793.

45 Kukida M, Mogi M, Kan-No H, Tsukuda K, Bai H-Y, Shan B-S, et al. AT2 receptor stimulation inhibits phosphate-induced vascular calcification. Kidney Int. 2019 Jan;95:138-48.

46 Kitagawa $M$, Sugiyama $H$, Morinaga $H$, Inoue $\mathrm{T}$, Takiue K, Ogawa A, et al. A decreased level of serum soluble Klotho is an independent biomarker associated with arterial stiffness in patients with chronic kidney disease. PLoS One. 2013;8(2):e56695.

47 Fan Q. Decreased level of serum soluble Klotho is a biomarker associated with vascular calcification in peritoneal dialysis patients. Hong Kong J Nephrol. 2015;17(2):S125-6. 
$48 \mathrm{Hu}$ MC, Shi M, Zhang J, Quiñones H, Griffith C, Kuro-o M, et al. Klotho deficiency causes vascular calcification in chronic kidney disease. J Am Soc Nephrol. 2011 Jan;22(1):12436.

49 Lim K, Lu TS, Molostvov G, Lee C, Lam FT, Zehnder D, et al. Vascular Klotho deficiency potentiates the development of human artery calcification and mediates resistance to fibroblast growth factor 23. Circulation. 2012 May 8;125(18):2243-55.

50 Morishita K, Shirai A, Kubota M, Katakura Y, Nabeshima Y, Takeshige K, et al. The progression of aging in klotho mutant mice can be modified by dietary phosphorus and zinc. J Nutr. 2001 Dec;131(12):3182-8.

51 Ohnishi M, Razzaque MS. Dietary and genetic evidence for phosphate toxicity accelerating mammalian aging. FASEB J. 2010 Sep; 24(9):3562-71.

52 Ohnishi M, Nakatani T, Lanske B, Razzaque MS. Reversal of mineral ion homeostasis and soft-tissue calcification of klotho knockout mice by deletion of vitamin D 1alpha-hydroxylase. Kidney Int. 2009 Jun;75(11):1166-72.

53 Nicoll R, Howard JM, Henein MY. A review of the effect of diet on cardiovascular calcification. Int J Mol Sci. 2015 Apr 21;16(4):8861-83.

54 Hum JM, O’Bryan LM, Tatiparthi AK, Cass TA, Clinkenbeard EL, Cramer MS, et al. Chronic hyperphosphatemia and vascular calcification are reduced by stable delivery of soluble Klotho. J Am Soc Nephrol. 2017 Apr; 28(4):1162-74.

55 Hanazaki A, Ikuta K, Sasaki S, Sasaki S, Koike $\mathrm{M}$, Tanifuji K, et al. Role of sodium-dependent $\mathrm{Pi}$ transporter/Npt2c on Pi homeostasis in klotho knockout mice different properties between juvenile and adult stages. Physiol Rep. 2020 Feb;8(3):e14324.

56 Liu L, Liu Y, Zhang Y, Bi X, Nie L, Liu C, et al. High phosphate-induced downregulation of PPAR $\gamma$ contributes to CKD-associated vascular calcification. J Mol Cell Cardiol. 2018 Jan; 114:264-75.

57 Duni A, Liakopoulos V, Roumeliotis S, Peschos D, Dounousi E. Oxidative stress in the pathogenesis and evolution of chronic kidney disease: untangling Ariadne's thread. Int J Mol Sci. 2019 Jul 29;20(15):3711.

58 Wang Y, Kuro-o M, Sun Z. Klotho gene delivery suppresses Nox 2 expression and attenuates oxidative stress in rat aortic smooth muscle cells via the cAMP-PKA pathway. Aging Cell. 2012 Jun;11(3):410-7.

59 Sun H, Zhang F, Xu Y, Sun S, Wang H, Du Q, et al. Salusin- $\beta$ promotes vascular calcification via nicotinamide adenine dinucleotide phosphate/reactive oxygen species-mediated klotho downregulation. Antioxid Redox Signal. 2019 Dec 20;31(18):1352-70.

60 Mizusaki K, Hasuike Y, Kimura T, Nagasawa Y, Kuragano T, Yamada Y, et al. Inhibition of the mammalian target of rapamycin may augment the increase in soluble klotho levels in renal transplantation recipients. Blood Purif. 2019;47(Suppl 2):12-8.
61 Chang JR, Guo J, Wang Y, Hou YL, Lu WW, Zhang JS, et al. Intermedin1-53 attenuates vascular calcification in rats with chronic kidney disease by upregulation of $\alpha$-Klotho. Kidney Int. 2016 Mar;89(3):586-600.

62 Di Lullo L, Gorini A, Russo D, Santoboni A Ronco C. Left ventricular hypertrophy in chronic kidney disease patients: from pathophysiology to treatment. Cardiorenal Med. 2015;5(4):254-66.

63 Grande D, Gioia MI, Terlizzese P, Iacoviello M. Heart failure and kidney disease. Adv Exp Med Biol. 2018;1067:219-38.

64 Zhang A-H, Guo W-K, Yu L, Liu W-H. Relationship of serum soluble klotho levels and echocardiographic parameters in patients on maintenance hemodialysis. Kidney Blood Press Res. 2019;44(3):396-404.

65 Xie J, Wu YL, Huang CL. Deficiency of soluble $\alpha$-Klotho as an independent cause of uremic cardiomyopathy. Vitam Horm. 2016;101: 311-30.

66 Tang G, Shen Y, Gao P, Song S-S, Si L-Y. Klotho attenuates isoproterenol-induced hypertrophic response in $\mathrm{H} 9 \mathrm{C} 2$ cells by activating $\mathrm{Na} / \mathrm{K}$-ATPase and inhibiting the reverse mode of $\mathrm{Na} / \mathrm{Ca}$-exchanger. In Vitro Cell Dev Biol Anim. 2018 Mar;54(3):250-6

67 Ding J, Tang Q, Luo B, Zhang L, Lin L, Han L, et al. Klotho inhibits angiotensin II-induced cardiac hypertrophy, fibrosis, and dysfunction in mice through suppression of transforming growth factor- $\beta 1$ signaling pathway. Eur J Pharmacol. 2019 Sep 15;859:172549.

68 Guo Y, Zhuang X, Huang Z, Zou J, Yang D, $\mathrm{Hu} \mathrm{X}$, et al. Klotho protects the heart from hyperglycemia-induced injury by inactivating ROS and NF- $\kappa \mathrm{B}$-mediated inflammation both in vitro and in vivo. Biochim Biophys Acta. 2018 Jan; 1864(1):238-51.

69 Razzaque MS. The FGF23-Klotho axis: endocrine regulation of phosphate homeostasis. Nat Rev Endocrinol. 2009;5(11):611-9.

70 Faul C, Amaral AP, Oskouei B, Hu MC, Sloan A, Isakova T, et al. FGF23 induces left ventricular hypertrophy. J Clin Invest. 2011 Nov; 121(11):4393-408

71 Leifheit-Nestler M, Große Siemer R, Flasbart K, Richter B, Kirchhoff F, Ziegler WH, et al. Induction of cardiac FGF23/FGFR4 expression is associated with left ventricular hypertrophy in patients with chronic kidney disease. Nephrol Dial Transplant. 2016 Jul;31(7): 1088-99.

72 Han X, Cai C, Xiao Z, Quarles LD. FGF23 induced left ventricular hypertrophy mediated by FGFR4 signaling in the myocardium is attenuated by soluble Klotho in mice. J Mol Cell Cardiol. 2020 Jan;138:66-74.

73 Suassuna PGdA, Cherem PM, de Castro BB, Maquigussa E, Cenedeze MA, Lovisi JCM, et al. aKlotho attenuates cardiac hypertrophy and increases myocardial fibroblast growth factor 21 expression in uremic rats. Exp Biol Med. 2020 Jan;245(1):66-78.

74 Xie J, Cha SK, An SW, Kuro-O M, Birnbaumer L, Huang CL. Cardioprotection by
Klotho through downregulation of TRPC6 channels in the mouse heart. Nat Commun. 2012;3:1238.

75 Xie J, Yoon J, An SW, Kuro-o M, Huang CL. Soluble Klotho protects against uremic cardiomyopathy independently of fibroblast growth factor 23 and phosphate. J Am Soc Nephrol. 2015 May;26(5):1150-60.

76 Paterson MR, Geurts AM, Kriegel AJ. miR146b-5p has a sex-specific role in renal and cardiac pathology in a rat model of chronic kidney disease. Kidney Int. 2019 Dec;96(6): 1332-45.

77 Wang B, Zhang A, Wang H, Klein JD, Tan L, Wang Z-M, et al. miR-26a limits muscle wasting and cardiac fibrosis through exosomemediated microRNA transfer in chronic kidney disease. Theranostics. 2019;9(7):1864-77.

78 Liu Q, Zhu L-J, Waaga-Gasser AM, Ding Y, Cao M, Jadhav SJ, et al. The axis of local cardiac endogenous Klotho-TGF- $\beta 1-W n t$ signaling mediates cardiac fibrosis in human. J Mol Cell Cardiol. 2019 Nov;136:113-24.

79 Libby P, Buring JE, Badimon L, Hansson GK, Deanfield J, Bittencourt MS, et al. Atherosclerosis. Nat Rev Dis Primers. 2019;5(1):56.

80 Drüeke TB, Massy ZA. Atherosclerosis in CKD: differences from the general population. Nat Rev Nephrol. 2010;6(12):723-35.

81 Keles N, Caliskan M, Dogan B, Keles NN, Kalcik M, Aksu F, et al. Low serum level of klotho is an early predictor of atherosclerosis. Tohoku J Exp Med. 2015 Sep;237(1):17-23.

82 Martín-Núñez E, Donate-Correa J, Ferri C, López-Castillo Á, Delgado-Molinos A, Hernández-Carballo C, et al. Association between serum levels of Klotho and inflammatory cytokines in cardiovascular disease: a case-control study. Aging (Albany NY). 2020 Jan 27;12(2): 1952-64.

83 Kuro-O M. The Klotho proteins in health and disease. Nat Rev Nephrol. 2019 Jan;15(1):2744.

84 Kim HY, Yoo TH, Hwang Y, Lee GH, Kim B, Jang J, et al. Indoxyl sulfate (IS)-mediated immune dysfunction provokes endothelial damage in patients with end-stage renal disease (ESRD). Sci Rep. 2017 Jun 8;7(1):3057.

85 Ohta J, Rakugi H, Ishikawa K, Yang J, Ikushima M, Chihara Y, et al. Klotho gene delivery suppresses oxidative stress in vivo. Geriatr Gerontol Int. 2007;7(3):293-9.

86 Rakugi H, Matsukawa N, Ishikawa K, Yang J, Imai M, Ikushima $\mathrm{M}$, et al. Anti-oxidative effect of Klotho on endothelial cells through cAMP activation. Endocrine. 2007 Feb;31(1): 82-7.

87 Cui W, Leng B, Wang G. Klotho protein inhibits $\mathrm{H}_{2} \mathrm{O}_{2}$-induced oxidative injury in endothelial cells via regulation of PI3K/AKT/ Nrf2/HO-1 pathways. Can J Physiol Pharmacol. 2019;97(5):370-6.

88 Yao Y, Wang Y, Zhang Y, Liu C. Klotho ameliorates oxidized low density lipoprotein (oxLDL)-induced oxidative stress via regulating LOX-1 and PI3K/Akt/eNOS pathways. Lipids Health Dis. 2017;16(1):77. 
89 Ikushima M, Rakugi H, Ishikawa K, Maekawa Y, Yamamoto K, Ohta J, et al. Anti-apoptotic and anti-senescence effects of Klotho on vascular endothelial cells. Biochem Biophys Res Commun. 2006;339(3):827-32.

90 Liu Y, Lai P, Deng J, Hao Q, Li X, Yang M, et al. sKlotho Micro-RNA335-5p targeted inhibition of and promoted oxidative stress-mediated aging of endothelial cells. Biomark Med. 2019 Apr;13(6):457-66.

91 Uhrin P, Wang D, Mocan A, Waltenberger B, Breuss JM, Tewari D, et al. Vascular smooth muscle cell proliferation as a therapeutic target. Part 2: natural products inhibiting proliferation. Biotechnol Adv. 2018;36(6):1608-21.

$92 \mathrm{Yu} \mathrm{S}$, Chen Y, Chen S, Ye N, Li Y, Sun Y. Klotho inhibits proliferation and migration of angiotensin II-induced vascular smooth muscle cells (VSMCs) by modulating NF- $\kappa B$ p65, Akt, and extracellular signal regulated kinase (ERK) signaling activities. Med Sci Monit. 2018 Jul 13;24:4851-60.

93 Maltese G, Psefteli PM, Rizzo B, Srivastava S, Gnudi L, Mann GE, et al. The anti-ageing hormone klotho induces Nrf2-mediated antioxidant defences in human aortic smooth muscle cells. J Cell Mol Med. 2017 Mar;21(3):621-7.

94 He T, Xiong J, Huang Y, Zheng C, Liu Y, Bi X, et al. Klotho restrain RIG-1/NF- $\kappa B$ signaling activation and monocyte inflammatory factor release under uremic condition. Life Sci. 2019 Aug 15;231:116570.

95 Jia Y, Zheng Z, Xue M, Zhang S, Hu F, Li Y, et al. Extracellular vesicles from albumin-induced tubular epithelial cells promote the M1 macrophage phenotype by targeting klotho. Mol Ther. 2019 Aug 7;27(8):1452-66.
96 Liu W, Chen X, Wu M, Li L, Liu J, Shi J, et al. Recombinant Klotho protein enhances cholesterol efflux of THP-1 macrophage-derived foam cells via suppressing $\mathrm{Wnt} / \beta$ catenin signaling pathway. BMC Cardiovasc Disord. 2020 Mar 5;20(1):120.

97 André P, Denis CV, Ware J, Saffaripour S, Hynes RO, Ruggeri ZM, et al. Platelets adhere to and translocate on von Willebrand factor presented by endothelium in stimulated veins. Blood. 2000 Nov 15;96(10): 3322-8.

98 Huo Y, Schober A, Forlow SB, Smith DF, Hyman MC, Jung $S$, et al. Circulating activated platelets exacerbate atherosclerosis in mice deficient in apolipoprotein E. Nat Med. 2003 Jan;9(1):61-7.

99 Liu M, Li XC, Lu L, Cao Y, Sun RR, Chen S, et al. Cardiovascular disease and its relationship with chronic kidney disease. Eur Rev Med Pharmacol Sci. 2014 Oct;18(19):2918-26.

100 Matsushita K, Ballew SH, Coresh J. Cardiovascular risk prediction in people with chronic kidney disease. Curr Opin Nephrol Hypertens. 2016;25(6):518-23.

101 Marçais C, Maucort-Boulch D, Drai J, Dantony E, Carlier MC, Blond E, et al. Circulating Klotho associates with cardiovascular morbidity and mortality during hemodialysis. J Clin Endocrinol Metab. 2017 Sep 1; 102(9):3154-61.

102 Memmos E, Sarafidis P, Pateinakis P, Tsiantoulas A, Faitatzidou D, Giamalis P, et al. Soluble Klotho is associated with mortality and cardiovascular events in hemodialysis. BMC Nephrol. 2019 Jun 11;20(1):217.

103 Yang K, Yang J, Bi X, Yu Z, Xiao T, Huang Y, et al. Serum klotho, cardiovascular events, and mortality in nondiabetic chronic kidney disease. Cardiorenal Med. 2020;10(3):175-87.

104 Zhang R, Zheng F. PPAR-gamma and aging: one link through klotho? Kidney Int. 2008 Sep;74(6):702-4.
105 Forster RE, Jurutka PW, Hsieh JC, Haussler CA, Lowmiller CL, Kaneko I, et al. Vitamin $D$ receptor controls expression of the antiaging klotho gene in mouse and human renal cells. Biochem Biophys Res Commun. 2011 Oct 28;414(3):557-62.

106 Yoon HE, Ghee JY, Piao S, Song JH, Han DH, Kim S, et al. Angiotensin II blockade upregulates the expression of Klotho, the anti-ageing gene, in an experimental model of chronic cyclosporine nephropathy. Nephrol Dial Transplant. 2011 Mar;26(3):800-13.

107 Ohnishi M, Kato S, Akiyoshi J, Atfi A, Razzaque MS. Dietary and genetic evidence for enhancing glucose metabolism and reducing obesity by inhibiting klotho functions. FASEB J. 2011 Jun;25(6):2031-9.

108 Hasannejad M, Samsamshariat SZ, Esmaili A, Jahanian-Najafabadi A. Klotho induces insulin resistance possibly through interference with GLUT4 translocation and activation of Akt, GSK3 $\beta$, and PFKf $\beta 3$ in 3T3-L1 adipocyte cells. Res Pharm Sci. 2019 Aug; 14(4):369-77.

109 Lorenzi O, Veyrat-Durebex C, Wollheim $\mathrm{CB}$, Villemin P, Rohner-Jeanrenaud F, Zanchi A, et al. Evidence against a direct role of klotho in insulin resistance. Pflugers Arch. 2010 Feb;459(3):465-73.

110 Zhao Y, Zhao M, Cai Y, Zheng M, Sun W, Zhang S, et al. Mammalian target of rapamycin signaling inhibition ameliorates vascular calcification via Klotho upregulation. Kidney Int. 2015 June 10;88(4):711-21.

111 Saito Y, Nakamura T, Ohyama Y, Suzuki T, Iida A, Shiraki-Iida T, et al. In vivo klotho gene delivery protects against endothelial dysfunction in multiple risk factor syndrome. Biochem Biophys Res Commun. 2000 Sep 24;276(2):767-72. 